Research Article

\title{
Solvability of Some Integral Equations in Banach Space and Their Applications to the Theory of Viscoelasticity
}

\author{
Onur Alp İlhan \\ Faculty of Education, Erciyes University, 38039 Melikgazi Kayseri, Turkey \\ Correspondence should be addressed to Onur Alp İlhan, oailhan@erciyes.edu.tr \\ Received 3 September 2011; Accepted 27 February 2012 \\ Academic Editor: Ibrahim Sadek
}

Copyright (C) 2012 Onur Alp İlhan. This is an open access article distributed under the Creative Commons Attribution License, which permits unrestricted use, distribution, and reproduction in any medium, provided the original work is properly cited.

\begin{abstract}
An integral equation of Volterra type with additional compact operator in Banach space is considered. A special case is an integral equation of contact problem that arises in theory of viscoelasticity of mixed Fredholm and Volterra type with spectral parameter depending on time. In case the initial value of the parameter coincides with some isolated point of the spectrum of compact operator, the conditions of solvability are established.
\end{abstract}

\section{Introduction}

We consider in an arbitrary complex Banach space $\mathbf{B}$ the following integral equation:

$$
\int_{0}^{t} K(t, s) u(s) d s+A u(t)-\lambda(t) u(t)=f(t), \quad t \geq 0,
$$

where $u:[0,+\infty) \rightarrow \mathbf{B}$ is unknown function, $A: \mathbf{B} \rightarrow \mathbf{B}$ is a linear compact operator, $K: Q \rightarrow \mathbb{C}$ is the kernel, $f:[0,+\infty) \rightarrow \mathbf{B}$ is a given function, and $\lambda:[0,+\infty) \rightarrow \mathbb{C}$ is a function which we may interpret as spectral parameter. We indicated above by $Q$ the set

$$
Q=\left\{(t, s) \in \mathbb{R}^{2}: 0 \leq s \leq t<\infty\right\} .
$$


The main example is the following integral equation:

$$
\int_{0}^{t} K(t, s) u(x, s) d s+\int_{\Omega} R(x, y) u(y, t) d y-\lambda(t) u(x, t)=f(x, t),
$$

where $x \in \Omega$ and $t \geq 0$, which we consider in Banach spaces $\mathbf{B}=L_{p}(\Omega)$ or $\mathbf{B}=C(\bar{\Omega})$. We suppose that the set $\Omega \subset \mathbb{R}^{n}$ is measurable in Lebesgue sense. The equations of such type are known as partial integral equations and were first considered by Salam [1] (see also [2], and books [3, 4]). The equation (1.3) arises in the theory of viscoelasticity [5] (see also [6]). The kernels $K(t, s)$ and $R(x, y)$ are connected with some elastic creeping base and $\lambda(t)$ is the given value which describes the elastic properties of deformable body. We may refer also to work [7], where the more general integral equations in Hilbert space were considered. The main purpose of the present paper is to find conditions of solvability of (1.1) in case where $\lambda(0)$ coincides with some isolated pole of the resolvent $R_{\lambda}(A)=(A-\lambda I)^{-1}$.

\section{The Conditions for Solvability on the Spectrum}

We suppose that $\lambda(t)$ is a continuous function. Denote by $\Lambda(t)$ the range of the function $\lambda(s)$ on the interval $[0, t]$

$$
\Lambda(t)=\{\lambda(s): 0 \leq s \leq t\}
$$

It is clear that because of continuity of the function $\lambda(t)$ the set $\Lambda(t)$ for every $t \geq 0$ is closed. We say that $u \in C_{\mathbf{B}}[0, \infty)$ if $u$ is an abstract function $u:[0,+\infty) \rightarrow \mathbf{B}$, which is continuous on the half-line $[0, \infty)$, and set

$$
\|u\|_{t}=\sup _{0 \leq s \leq t}\|u(s)\|, \quad t \geq 0
$$

Denote by $\sigma(A)$ the spectrum of a compact operator $A$ and consider for $\lambda \notin \sigma(A)$ the resolvent $R_{\lambda}(A)=(A-\lambda I)^{-1}$ of the operator $A$. In case where $\Lambda(t) \cap \sigma(A)=\oslash$ for all $t \geq 0$ it is not difficult to show that (1.1) has continuous solution $u(t)$ for any continuous function $f(t)$. The problem is more complicated when $\Lambda(t)$ has a common point with spectrum of $A$ and that is the main idea of our consideration. Note that the case where $A$ is self-adjoint operator in Hilbert space was studied in [7-9]. In this paper we assume that $\lambda(0)$ coincides with one of the points $\lambda_{0} \neq 0$ of the spectrum of the operator $A$. From the mechanical point of view it means that the initial state of the considered system coincides with resonance. The problem is how to change the function $\lambda(t)$ for $t>0$ in order to get the existence and uniqueness of the solution. It was proved in [7] that the answer is almost obvious: $\lambda(t)$ must go away from the spectrum as fast as possible. We suppose that $\lambda(t)$ has $m$ continuous derivatives on the half line $t \geq 0$, where $m$ will be chosen below. The main assumption is the following:

$$
\lambda^{\prime}(0) \neq 0
$$


It is necessary to add some conditions to establish the one-valued solvability of (1.1). It was shown in [7] that one of these conditions is $K(0,0)=0$. Indeed, if $K(0,0) \neq 0$ then there exists the function $\lambda(t)$, which satisfies condition (2.3), however the homogeneous equation

$$
\int_{0}^{t} K(t, s) u(s) d s+[A-\lambda(t) I] u(t)=0
$$

has nontrivial solution.

\subsection{Example}

Let $\mu \neq 0$ be a simple eigenvalue of the operator $A$ and $u_{\mu}$ be the corresponding eigenvector:

$$
A u_{\mu}=\mu u_{\mu}
$$

$$
\begin{aligned}
& \text { Set } u(t)=u_{\mu}=\text { const } \neq 0 \text {. Then } \\
& \qquad \int_{0}^{t} K(t, s) u(s) d s+[A-\lambda(t) I] u(t)=[k(t)+\mu-\lambda(t)] u_{\mu},
\end{aligned}
$$

where

$$
k(t)=\int_{0}^{t} K(t, s) d s
$$

Set

$$
\lambda(t)=\mu+k(t) .
$$

In case where $K(0,0) \neq 0$ this function satisfies condition (2.3) since

$$
\lambda^{\prime}(0)=K(0,0) \neq 0 .
$$

It is clear that $u(t)$ is a solution of the homogeneous equation (2.4).

Let $p$ be a natural number. We assume that the kernel $K(t, s)$ is defined on the whole plane $\mathbb{R}^{2}$, has all partial derivatives of order $\leq p$, which are continuous on the plane $\mathbb{R}^{2}$ and the following conditions:

$$
\frac{\partial^{i+j} K}{\partial t^{i} \partial s^{j}}(0,0)=0, \quad 0 \leq i+j \leq p-1
$$

are fulfilled. For example, $K(t, s)=(t \pm s)^{p} F(t, s)$, where $F(t, s)$ is an arbitrary smooth enough function on the plane $\mathbb{R}^{2}$. 
Let $A^{*}: \mathbf{B}^{*} \rightarrow \mathbf{B}^{*}$ be the adjoint operator, where $\mathbf{B}^{*}$ is a conjugate space (the space of linear continuous functionals $v: \mathbf{B} \rightarrow \mathbb{C}$ ). As usual, we set

$$
\operatorname{ker} A^{*}=\left\{v \in \mathbf{B}^{*}: A^{*} v=0\right\} .
$$

Further, we say that $u \perp \operatorname{ker} A^{*}$ if $u \in \mathbf{B}$ and $v(u)=0$ for all $v \in \operatorname{ker} A^{*}$.

According to Fredholm-Riesz-Schauder theory, every nonzero point $\lambda_{0}$ of the spectrum of $A$ is an eigenvalue and

$$
\operatorname{dim} \operatorname{ker}\left(A-\lambda_{0} I\right)=\operatorname{dim} \operatorname{ker}\left(A^{*}-\lambda_{0} I\right) \geq 1
$$

It is well known that the resolvent of the compact operator is meromorphic function and the nonzero eigenvalues of this operator coincide with poles of this function (see, e.g., [10], Chapter VIII, Section 8).

\section{Solvability on the Spectrum}

Definition 3.1. Let $m$ be a natural number and $\lambda_{0}$ be an eigenvalue of the compact linear operator $A$. We say that $\lambda_{0} \neq 0$ is the isolated point of the spectrum of the operator $A$ of the type $m$ if $\lambda_{0}$ is the pole of the resolvent $R_{\lambda}(A)=(A-\lambda I)^{-1}$ of the order $m$, that is, there exist $C>0$ and $\delta>0$ so that

$$
\left\|R_{\lambda}(A)\right\| \leq \frac{C}{\left|\lambda-\lambda_{0}\right|^{m}}, \quad 0<\left|\lambda-\lambda_{0}\right|<\delta
$$

We apply also the additional restrictions on the function $f$ and its derivatives at the point $t=0$ and prove the following result. In what follows we assume that the condition (2.10) with some natural $p$ is fulfilled.

We say that the function $\lambda:[0,+\infty) \rightarrow \mathbb{C}$ belongs to the space $C^{m}[0, \infty)$, if this function has $m$ continuous derivatives for $t \geq 0$.

Theorem 3.2. Suppose that $\lambda \in C^{m}[0, \infty)$ satisfies condition (2.3). Let $\lambda(0)$ be an isolated point of the spectrum $\sigma(A)$ of the type $m \leq p$ and $\lambda(t) \notin \sigma(A)$ for all $t>0$. If the function $f$ has continuous derivatives of order $\leq m$ on the half-line $t \geq 0$, and the following conditions:

$$
f^{(k)}(0) \perp \operatorname{ker}\left(A^{*}-\lambda_{0} I\right), \quad k=0,1, \ldots, m-1,
$$

are fulfilled, then the continuous solution of (1.1) exists and is unique.

To prove Theorem 3.2 we consider the following auxiliary equation:

$$
\int_{0}^{t} K(t, s) R_{\lambda(s)}(A) v(s) d s+v(t)=f(t), \quad t \geq 0,
$$

which is equivalent to (1.1). At first we find the estimate of resolvent $R_{\lambda(t)}$ near to point $\lambda_{0}=\lambda(0)$. 
Lemma 3.3. Let $\lambda(0)=\lambda_{0}$ be an isolated point of the spectrum $\sigma(A)$ of the type $m$ and $\lambda(t) \notin \sigma(A)$ for all $t>0$. If the condition (2.3) is fulfilled then for all $T>0$ the following inequality:

$$
\left\|R_{\mathcal{\lambda}(t)}(A)\right\| \leq \frac{C_{T}}{t^{m}}, \quad 0<t \leq T,
$$

is valid.

Proof. It is not difficult to show that the estimate

$$
\left|\lambda(t)-\lambda_{0}\right| \geq c(T) t, \quad 0 \leq t \leq T
$$

follows from assumption (2.3). According to condition (3.1), for all $\lambda$ near to $\lambda_{0}$ the estimate

$$
\left\|R_{\lambda(t)}(A)\right\| \leq \frac{C}{\left|\lambda(t)-\lambda_{0}\right|^{m}}
$$

is valid, and (3.4) follows from (3.5) and (3.6).

Lemma 3.4. Let the conditions (2.10) be fulfilled. Then for $m \leq p$ the following equality

$$
K(t, s)=t^{m} K_{m}(t, s)
$$

is valid, where the function $K_{m}(t, s)$ is bounded on every compact set $E \subset Q$.

Proof. The assertion follows from equality

$$
\begin{aligned}
K(t, s) & =\sum_{i+j \leq m} \frac{1}{i ! j !} \frac{\partial^{i+j} K(0,0)}{\partial t^{i} \partial s^{j}} t^{i} s^{j}+R_{m}(t, s) \\
& =\sum_{i+j=m} \frac{1}{i ! j !} \frac{\partial^{i+j} K(0,0)}{\partial t^{i} \partial s^{j}} t^{i} s^{j}+R_{m}(t, s)=t^{m} K_{m}(t, s) .
\end{aligned}
$$

We are taking into account that $0 \leq s \leq t$ and, hence,

$$
t^{i} s^{j}=t^{m} \cdot\left(\frac{s}{t}\right)^{j}=O\left(t^{m}\right), \quad i+j=m
$$

Lemma 3.5. Let the function $g$ be continuous on the open half-line $(0,+\infty)$ and bounded on the closed half-line $[0,+\infty)$. Then for every $T>0$ the solution of the equation

$$
\int_{0}^{t}\left(\frac{s}{t}\right)^{m} K(t, s) R_{\lambda(s)}(A) w(s) d s+w(t)=g(t), \quad 0 \leq t \leq T,
$$

exists, is unique, continuous, and bounded on the interval $(0, T]$. 
Proof. According to Lemma 3.4, we may state that the function

$$
K_{m}(t, s)=t^{-m} K(t, s)
$$

is bounded for $0 \leq s \leq t \leq T$. Set

$$
B(t)=t^{m} R_{\lambda(t)}(A) \text { for } t>0, B(0)=0 .
$$

Then, (3.10) takes the form

$$
\int_{0}^{t} K_{m}(t, s) B(s) w(s) d s+w(t)=g(t), \quad 0 \leq t \leq T .
$$

Note that, according to Lemma 3.3,

$$
\|B(t) w(t)\| \leq C\|w(t)\|, \quad 0 \leq t \leq T .
$$

Now it is clear that the integral operator on the left side (3.13) is quasi-nilpotent. Hence (3.13) has required unique solution, which is given by Neumann series.

Lemma 3.6. Let $f(t)=t^{m} g(t)$, where the function $g$ is continuous on the half-line $[0, \infty)$. Then the continuous solution of (1.1) exists and is unique.

Proof. Set

$$
v(t)=t^{m} w(t)
$$

where $w(t)$ is a solution to (3.10). Then $v(t)$ is a solution to (3.3). Hence, the function $u(t)=$ $R_{\lambda(t)}(A) v(t)$ is the required solution of (1.1).

For an arbitrary $T>0$ this solution exists on the interval $[0, T]$, and because of uniqueness we may state that this solution belongs to $C_{\mathbf{B}}[0, \infty)$.

Lemma 3.7. Let $f_{k} \in \mathbf{B}$ and

$$
f_{k} \perp \operatorname{ker}\left(A^{*}-\lambda_{0} I\right), \quad k=0,1, \ldots, m-1 .
$$

Then there exist the elements $\theta_{k j} \in \mathbf{B}, 0 \leq k \leq m-1,0 \leq j \leq m-k-1$, so that

$$
\theta_{k j} \perp \operatorname{ker}\left(A^{*}-\lambda_{0} I\right)
$$

and the functions

$$
v_{k}(t)=t^{k}\left(\theta_{k 0}+t \theta_{k 1}+t^{2} \theta_{k 2}+\cdots+t^{m-k-1} \theta_{k, m-k-1}\right)
$$


which satisfy the following equations:

$$
[A-\lambda(t) I] v_{k}(t)=t^{k} f_{k}+t^{m} \beta_{k}(t)
$$

where the functions $\beta_{k}(t)$ are continuous on the half-line $[0,+\infty)$ and

$$
\beta_{k}(t) \perp \operatorname{ker}\left(A^{*}-\lambda_{0} I\right), \quad t \geq 0 .
$$

Proof. We construct the elements $\theta_{k j}$ using the back induction and begin with the case $k=$ $m-1$. It is clear that we may choose element $\theta_{m-1} \perp \operatorname{ker}\left(A^{*}-\lambda_{0} I\right)$ so that

$$
\left[A-\lambda_{0} I\right] \theta_{m-1}=f_{m-1}
$$

Set

$$
v_{m-1}(t)=t^{m-1} \theta_{m-1}
$$

Then

$$
\begin{aligned}
{[A-\lambda(t) I] v_{m-1}(t) } & =\left[A-\lambda_{0} I\right] v_{m-1}(t)-\left[\lambda(t)-\lambda_{0}\right] v_{m-1}(t) \\
& =t^{m-1}\left[A-\lambda_{0} I\right] \theta_{m-1}-\left[\lambda(t)-\lambda_{0}\right] t^{m-1} \theta_{m-1} \\
& =t^{m-1} f_{m-1}+t^{m} \beta_{m-1}(t),
\end{aligned}
$$

where

$$
\begin{gathered}
\beta_{m-1}(t)=-\frac{\lambda(t)-\lambda_{0}}{t} \theta_{m-1}, \quad t>0 \\
\beta_{m-1}(0)=-\lambda^{\prime}(0) \theta_{m-1} .
\end{gathered}
$$

It is clear that $\beta_{m-1}(t)$ satisfies condition (3.20).

Now assume that Lemma 3.3 is valid for some $k<m$ and prove it for $k-1$, that is, show that there exist $\theta_{k-1, j}$ so that the function

$$
v_{k-1}(t)=t^{k-1}\left(\theta_{k-1,0}+t \theta_{k-1,1}+t^{2} \theta_{k-1,2}+\cdots+t^{m-k} \theta_{k-1, k}\right)
$$

satisfies equation

$$
[A-\lambda(t) I] v_{k-1}(t)=t^{k-1} f_{k-1}+t^{m} \beta_{k-1}(t) .
$$


If we change $f_{k}$ to $f_{k-1}(3.18)$ then, we may state that there exists the function $u_{k}(t)$ so that

$$
\begin{gathered}
u_{k}(t)=t^{k}\left(\tilde{\theta}_{k 0}+t \tilde{\theta}_{k 1}+t^{2} \tilde{\theta}_{k 2}+\cdots+t^{m-k-1} \tilde{\theta}_{k, m-k-1}\right), \\
{[A-\lambda(t) I] u_{k}(t)=t^{k} f_{k-1}+t^{m} \tilde{\beta}_{k}(t) .}
\end{gathered}
$$

Set for $t \geq 0$

$$
v_{k-1}(t)=t^{k-1}\left(\tilde{\theta}_{k 0}+t \tilde{\theta}_{k 1}+t^{2} \tilde{\theta}_{k 2}+\cdots+t^{m-k-1} \tilde{\theta}_{k, m-k-1}+t^{m-k} \theta^{*}\right)
$$

where $\theta^{*}$ will be defined below. Taking into account (3.27) we may write for $t>0$

$$
v_{k-1}(t)=\frac{1}{t} u_{k}(t)+t^{m-1} \theta^{*}
$$

Then, according to (3.28),

$$
\begin{aligned}
{[A-\lambda(t) I] v_{k-1}(t) } & =\frac{1}{t}[A-\lambda(t) I] u_{k}(t)+t^{m-1}[A-\lambda(t) I] \theta^{*} \\
& =t^{k-1} f_{k-1}+t^{m-1} \tilde{\beta}_{k}(t)+t^{m-1}[A-\lambda(t) I] \theta^{*} \\
& =t^{k-1} f_{k-1}+t^{m-1} \tilde{\beta}_{k}(t)+t^{m-1}\left[A-\lambda_{0} I\right] \theta^{*}-t^{m-1}\left[\lambda(t)-\lambda_{0}\right] \theta^{*}
\end{aligned}
$$

If we choose $\theta^{*} \perp \operatorname{ker}\left(A^{*}-\lambda_{0} I\right)$ so that

$$
\left(A-\lambda_{0} I\right) \theta^{*}=-\tilde{\beta}_{k}(0),
$$

then

$$
\begin{aligned}
{[A-\lambda(t) I] v_{k-1}(t) } & =t^{k-1} f_{k-1}+t^{m-1}\left[\tilde{\beta}_{k}(t)-\tilde{\beta}_{k}(0)\right]-t^{m-1}\left[\lambda(t)-\lambda_{0}\right] \theta^{*} \\
& =t^{k-1} f_{k-1}+t^{m} \beta_{k-1}(t)
\end{aligned}
$$

where

$$
\begin{gathered}
\beta_{k-1}(t)=\frac{\tilde{\beta}_{k}(t)-\tilde{\beta}_{k}(0)}{t}-\frac{\lambda(t)-\lambda_{0}}{t} \theta^{*} \text { for } t>0, \\
\beta_{k-1}(0)=\tilde{\beta}_{k}^{\prime}(0)-\lambda^{\prime}(0) \theta^{*} .
\end{gathered}
$$

It is clear that the function $v_{k-1}(t)$ satisfies both conditions (3.25) and (3.26). 
Now, let us give the proof of Theorem 3.2.

Proof. Set

$$
f_{k}=\frac{f^{(k)}(0)}{k !}
$$

According to (3.2) these elements satisfy conditions (3.16) of Lemma 3.7. Let $v_{k}(t)$ be the solutions of (3.19) from Lemma 3.7. Set

$$
v(t)=\sum_{k=0}^{m-1} v_{k}(t)
$$

Then, according to Lemma 3.7,

$$
[A-\lambda(t) I] v(t)=\sum_{k=0}^{m-1} t^{k} \frac{f^{(k)}(0)}{k !}+t^{m} \beta(t)
$$

where $\beta(t) \in C[0, \infty)$. Hence,

$$
\int_{0}^{t} K(t, s) v(s) d s+[A-\lambda(t) I] v(t)=\sum_{k=0}^{m-1} \frac{f^{(k)}(0)}{k !} t^{k}+t^{m} h(t),
$$

where

$$
h(t)=\beta(t)+\int_{0}^{t} K_{m}(t, s) v(s) d s
$$

and the kernel $K_{m}(t, s)$ is defined by (3.7).

Set

$$
\tilde{f}(t)=f(t)-\int_{0}^{t} K(t, s) v(s) d s-[A-\lambda(t) I] v(t) .
$$

According to (3.38),

$$
\tilde{f}(t)=f(t)-\sum_{k=0}^{m-1} \frac{f^{(k)}(0)}{k !} t^{k}-t^{m} h(t)=t^{m} g(t),
$$

where, obviously, $g \in C[0, \infty)$ and

$$
g(0)=\frac{f^{(m)}(0)}{m !}-h(0)
$$


Consider the following equation:

$$
\int_{0}^{t} K(t, s) w(s) d s+[A-\lambda(t) I] w(t)=\tilde{f}(t)
$$

where $\tilde{f}(t)=t^{m} g(t)$. According to Lemma 3.6, the solution $w(t)$ exists and is unique.

Recall that according to definition (3.40), the function $v(t)$ satisfies equation

$$
\int_{0}^{t} K(t, s) v(s) d s+[A-\lambda(t)] v(t)=f(t)-\tilde{f}(t)
$$

Now we can prove the existence of the solution by setting

$$
u(t)=v(t)+w(t) .
$$

Uniqueness follows from Lemma 3.6.

\subsection{Remark}

Note that assumption (3.2) of Theorem 3.2 is important. If this is not satisfied then existence of the continuous solution is not guaranteed.

In order to show this, it is enough to consider the finite-dimensional operator with corresponding Jordan matrix whose diagonal and subdiagonal elements are equal to 1 and all other elements are equal to 0 . The next example makes clear this statement.

\subsection{Example}

Let us consider (1.3) for $\Omega=[0, \pi]$ and for kernel

$$
R(x, y)=\sum_{k=1}^{m} g_{k}(y) \sin k x, \quad 0 \leq x \leq \pi, 0 \leq y \leq \pi,
$$

where

$$
g_{k}(y)=\frac{2}{\pi}[\sin k y+\sin (k-1) y]
$$

Define the matrix $\left\|a_{j k}\right\|$ with elements

$$
a_{j k}=\int_{0}^{\pi} g_{j}(x) \sin k x d x .
$$


It is clear that

$$
a_{j j}=1, \quad j=1,2, \ldots, m, \quad a_{j(j-1)}=1, \quad j=2,3, \ldots, m,
$$

and $a_{j k}=0$ otherwise.

Let $u(x)$ be the solution to the equation

$$
(A-\lambda I) u(x)=f(x),
$$

or

$$
\mathcal{\jmath} u(x)=\sum_{k=1}^{m}\left(u, g_{k}\right) \sin k x-f(x),
$$

where, as usual,

$$
(u, v)=\int_{0}^{\pi} u(x) v(x) d x
$$

Set

$$
c_{k}=\left(u, g_{k}\right), \quad f_{k}=\left(f, g_{k}\right)
$$

Then (3.51) takes the form

$$
\lambda c_{j}=\sum_{k=1}^{m} a_{j k} c_{k}-f_{j}, \quad j=1,2, \ldots, m
$$

We suppose that $\lambda \neq 0$ and $\lambda \neq 1$. Then the solution of this algebraic system is

$$
c_{k}=-\sum_{j=1}^{k}(\lambda-1)^{j-k-1} f_{j}, \quad k=1,2, \ldots, m
$$

Hence, the solution of the integral equation (3.51) equals

$$
u(x)=\frac{1}{\lambda} \sum_{k=1}^{m} c_{k} \sin k x-\frac{1}{\ell} f(x) .
$$

Assume that

$$
f(x, t)=\varphi(t) \sin x .
$$


Then, obviously,

$$
\begin{gathered}
f_{1}(t)=f_{2}(t)=\varphi(t) \\
f_{3}=f_{4}=\cdots=f_{m}=0 .
\end{gathered}
$$

In this case, according to (3.55),

$$
c_{k}=-\frac{\varphi(t)}{(\lambda-1)^{k}}-\frac{\varphi(t)}{(\lambda-1)^{k-1}}=-\frac{\lambda}{(\lambda-1)^{k}} \varphi(t), \quad k=1,2, \ldots, m
$$

Thus, the function

$$
u(x, t)=-\varphi(t) \sum_{k=1}^{m} \frac{\sin k x}{(\lambda-1)^{k}}-\frac{\varphi(t)}{\lambda} \sin x
$$

satisfies (3.50) with right-hand side $f(x, t)$ defined by (3.57).

Now it is easy to construct (1.3) so that its right-hand side satisfies conditions (3.2) for $k=1,2, \ldots, m-2$ and does not satisfy this condition for $k=m-1$, and because of that this equation has no continuous solution.

For example, in the simplest case $K(t, s) \equiv 0$ we may set $\lambda(t)=1+t$ and $\varphi(t)=t^{m-1}$. The function (3.57) equals $f(x, t)=t^{m-1} \sin x$ and satisfies conditions (3.2) for all $k$ except $k=m-1$. According to (3.60), there exists only one solution

$$
u(x, t)=-\frac{1}{t} \sum_{k=1}^{m} t^{m-k} \sin k x-\frac{t^{m-1}}{1+t} \sin x
$$

and it is clear that this solution does not belong to $C[0, \infty)$.

\subsection{Remark}

We may note that the estimate of the solution may be obtained using the properties of some entire functions just as in [11].

\section{Acknowledgment}

The author is grateful to Professor Shavkat Alimov for his valuable assistance.

\section{References}

[1] A. Salam, "Fredholm solutions of partial integral equations," Mathematical Proceedings of the Cambridge Philosophical Society, vol. 49, pp. 213-217, 1953.

[2] S. Fenyo, "Beitrag zur Theorie der linearen partiellen Integralgleichungen," Publicationes Mathematicae Debrecen, vol. 4, pp. 98-103, 1955. 
[3] J. M. Appell, A. S. Kalitvin, and P. P. Zabrejko, Partial integral Operators and Integro-Differential Equations, vol. 230 of Monographs and Textbooks in Pure and Applied Mathematics, Marcel Dekker, New York, NY, USA, 2000.

[4] M. Väth, Volterra and Integral Equations of Vector Functions, vol. 224 of Monographs and Textbooks in Pure and Applied Mathematics, Marcel Dekker, New York, NY, USA, 2000.

[5] V. Volterra and E. Volterra, Sur les Distorsions des Corps Élastiques. Théorie et Applications, Mém. Sci. Math., Fasc. 147, Gauthier-Villars, Paris, France, 1960.

[6] Sh. A. Alimov and T. Sh. Shirinkulov, "Solution of contact problems in creep theory," Differential Equations, vol. 25, no. 9, pp. 1120-1124, 1989.

[7] S. Albeverio and S. Alimov, "On some integral equations in Hilbert space with an application to the theory of elasticity," Integral Equations and Operator Theory, vol. 55, no. 2, pp. 153-168, 2006.

[8] O. A. Ilhan, "Solvability of some integral equations in Hilbert spaces," Electronic Journal of Differential Equations, no. 116, article 7, 2004

[9] O. A. İlhan, "Solvability of some partial integral equations in Hilbert space," Communications on Pure and Applied Analysis, vol. 7, no. 4, pp. 837-844, 2008.

[10] K. Iosida, Functional Analysis, Springer, Berlin, Germany, 1965.

[11] S. A. Alimov and O. A. Ilhan, "An inequality associated with some entire functions," Journal of Inequalities in Pure and Applied Mathematics, vol. 5, no. 3, article 67, 2004. 


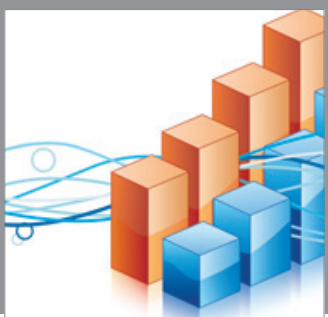

Advances in

Operations Research

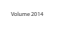

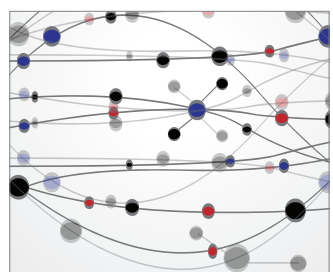

\section{The Scientific} World Journal
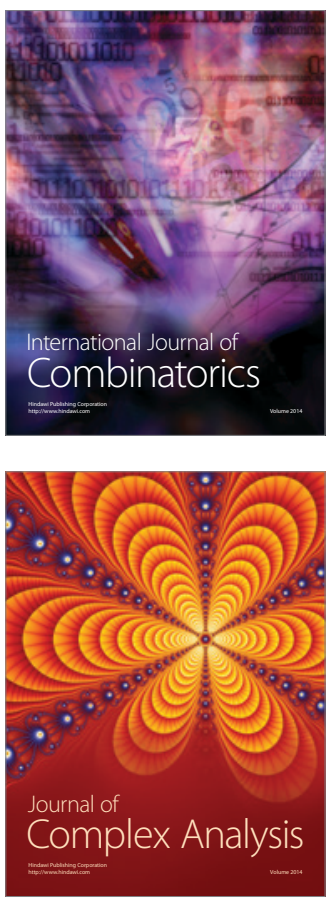

International Journal of

Mathematics and

Mathematical

Sciences
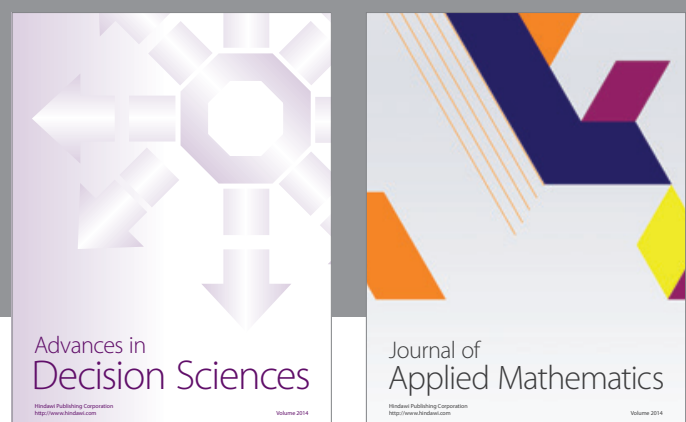

Journal of

Applied Mathematics
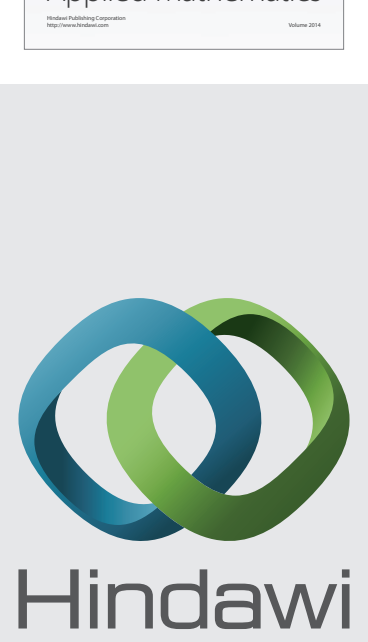

Submit your manuscripts at http://www.hindawi.com
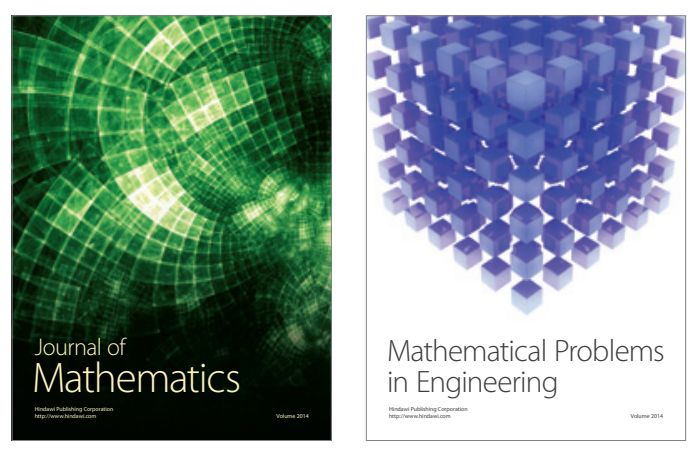

Mathematical Problems in Engineering
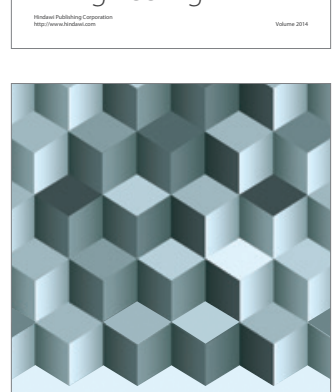

Journal of

Function Spaces
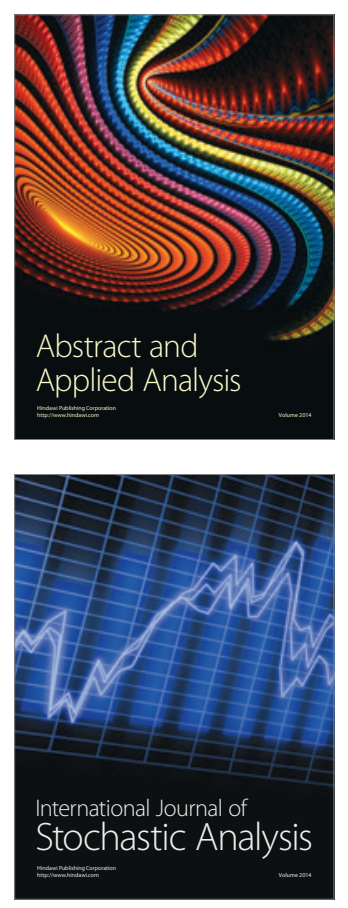

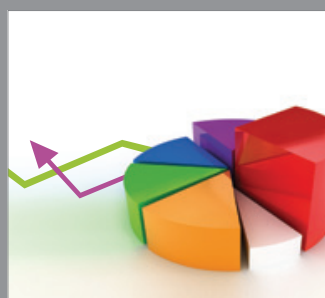

ournal of

Probability and Statistics

Promensencen
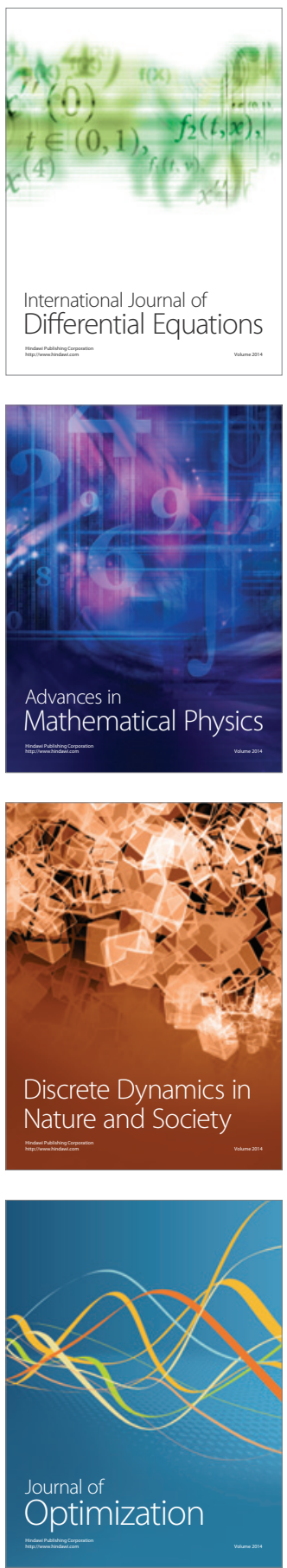